\title{
DETERMINACION DE METALES PESADOS BIOACUMULABLES EN ESPECIES ICTICAS DE CONSUMO HUMANO EN LA AMAZONIA PERUANA
}
R. Pezo D.*
H. Paredes A .*
N. Y . Bedayán $A .^{* *}$

\section{RESUMEN}

En el presente trabajo se reportan resultados de los análisis químicos realizados en 26 especies de peces de consumo humano, de los cuales 19 son de mayor demanda en la ciudad de Iquitos. Se han estudiado cuatro metales pesados bioacumulables como M ercurio, Cadmio, Plomo y Cobre.

Se han muestreado especies de tres ríos diferentes; Nanay, U cayali y A mazonas. Las concentraciones en su mayoría son menores que los límites establecidos intemacionalmente para pescado y productos pesqueros a excepción del "Dorado" (Brachyplatistoma fiavicans).

Palabras Claves: $\quad$ M etales, bioacumulables, peces, amazonía peruana.

\section{INTRODUCCION}

EI presente estudio, es la continuación de aquellos iniciados por el JIAP, en el monitoreo ambiental en las zonas de explotación petrolera, lugares donde se encontraron niveles superiores de concentración en especies ícticas de gran consumo por la población ribereña y citadina. Esto motivó que en la ciudad de Iquitos, lugar donde se comercializan los mayores volúmenes de pescado en diversas formas de presentación, se estudics los niveles de concentración en las especies de mayor consumo popular y de aquellos cuyo comportamiento de oferta permanece casi permanente durante el año, como es el caso del Dorado (Brachyplatistorna fiavicans).

Instituto de Investigaciones de la A mazonía Peruana (IIAP). A v. A belardo Quiñones

Km. 2.5. A partado 784. Iquitos - Perú

Facultad de Ciencias Biológicas. U niversidad Nacional de la A mazonía Peruana. 
Pevas 5ta. cdra. A partado 326. Iquitos - Perú.

Para satisfacción de los ambientalistas, del público en general así como para los autores, las concentraciones que se reportan están por debajo de los niveles máximos permitidos para el consumo humano directo.

\section{ASPECTOS GENERALES DE LOS METALES BIOACUMULABLES}

\section{Mercurio}

La formación del metil mercurio, que es la forma masiva atrajo la atención como contaminante, ambiental ocurrido en la Bahía de M inamata, Japón por vaciado de metil mercurio en un río y en una bahía, Jernelov (1975) menciona que antes que el mercurio llegue a convertirse en un contaminante peligroso en peces, moluscos o crustáceos, tiene que sufrir una metilación biológica, la cual es un proceso lento, si se compara con la acumulación biológica. Sin embargo, existen algunas condiciones que aumentan considerablemente la proporción de metilación biológica (Sic.).

El mismo autor menciona, que del total presente en un ecosistema acuático sólo una pequeña proporción se encuentra generalmente en forma metilada. Sin embargo, esta fracción es importante desde el punto de vista de la contaminación de peces ya que su vida media biológica en ellos puede ser medida en años mientras que del mercurio inorgánico sólo se refiere a semanas. Para el consumo humano, el metil mercurio es mucho más peligroso, al parecer existen dos formas principales en que puede aumentar la concentración en los organismos acuáticos especialmente en los peces:

Primero, por un aumento en la cantidad total de mercurio y, segundo debido al aumento en la proporción del mercurio total que existe en la forma de metil mercurio, lo cual podría resultar en un aumento de la cantidad de la metilación biológica de mercurio.

El mercurio derivado de la actividad humana, como de fuentes naturales, puede llegar al río por escurrimiento superficial y por la atmósfera a través de las precipitaciones pluviales. En el agua dulce se encuentra adherido a partículas de orígen orgánico e inorgánico. A hora bien el tamaño de las partículas puede variar desde muy pequeños. La habilidad para unirse al mercurio depende más de la superficie que de la masa, por lo que las partículas más pequeñas juegan un rol más importante debido a la presencia de especies iliófagos y filtradores. Como la mayor proporción de mercurio está unido a partículas orgánicas y éstos son metabolizados por microorganismos, el mercurio es eliminado a la forma metilada de tal manera que aumenta la proporción de metilación biológica del mercurio. 


\section{Transporte y Acumulución de Mercurio}

Los compuestos mercúricos volátiles, como el mercurio metálico y el dimetil mercurio se evaporan hacia la atmósfera. El uso de mercurio en las industrias, el quemar combustibles que contienen mercurio y el lavado de mineral es que contienen mercurio contribuyen a la contaminación atmosférica.

El dimetil mercurio es inestable en la atmósfera, en contacto con ácidos es delegado a la forma de monometil soluble en agua y por acción de la luz ultravioleta es descompuesto a mercurio (inorgánico) elemental (Sic)

\section{Contenido de Mercurio en los Organismos Acuáticos}

El planeta contiene frecuentemente niveles de mercurio de 2-10 ppm, la mayor parte en la forma inorgánica, mientras que en los peces varía desde 0.01 a 2 ppm, con una fracción significativa de metil mercurio unido a proténas no disuelto en grasa como es el caso de los hidrocarburos dorados. Consecuentemente el contenido de mercurio en los peces no depende de la presencia de grasa sino más bién de su nivel trófico, tamaí'io y edad. Esto nos indica que el mayor contenido de mercurio se encuentra en los grandes predadores.

El problema del mercurio es comúnmente considerado en relación al consumidor de peces y no como un problema en la producción de peces, salvo una toxicidad aguda, que puede producir una concentración de 20 p.p.m.

\section{Cadmio}

En aguas superficiales proviene de los efluentes de galvanoplastía; así como puede ser de origen alimentario, proviniendo de vasijas barnizadas y de utensilios de cocina gal vanizados.

En agua dulce, el cadmio puede estar frecuentemente unido a sustancias orgánicas, especialmente en plantas que contienen clorófila. Es posible que mamíferos acuáticos como las nutrias para el caso de los mismos contienen en sus rtnones hasta 500 p.p.m. de cadmio, estas concentraciones causarían serios daños renales en el hombre. Los efectos biológicos de estos niveles de cadmio en nutrias marinas y acuáticas, en sus organismos son desconocidos.

La dosis tóxica de cadmio por ingestión es de algunos miligramos, aproximadamente del 5 al $10 \%$ de lo absorbido es acumulado en los riñones bajo la forma de metalotionina, siendo su período biológico entre los 16 a 33 anos.

El nivel crítico en el córtex renal es de aproximadamente $200 \mathrm{mg} / \mathrm{kg}$. Situándose el nivel normal entre los 20 y $100 \mathrm{mg} / \mathrm{kg}$. Para un hombre de 50 anos. 
Para la vida acuática, en particular los peces, no parece que hubiera problemas por debajo de $1 \mathrm{mg} / \mathrm{l}$. sin embargo, las concentraciones elevadas pueden encontrarse en los moluscos y crustáceos de estuarios contaminados.

\section{Niveles Tróficos de las E species E studiadas}

En los especímenes del río Nanay se tiene 06 especies de iliófagos como Prochilodus nigricans, Potamorhina altamazónica, Curimatella sp, Hemiodus sp, Psectogaster amazónica, Potamorhina sp.; 03 especies de omnívoras con tendencia a frugívoras: Triportheus elongatus, Mylossoma aureum, Schizodotn sp.

Para el caso de los especímenes del río U cayali; 04 especies de iliófagos: Potamorhina altamazónica, Prochilodus nigricans, Hemiodus sp, Hypopthalinus edentatus;

07 especies de omnívoras: Brycon melanopterum, Piaractus brachypomum, Leporinos fasciatus, Colossoma macropomum, Mylossoma aureum, Schizodom fasciatus, Triporteus elongatus.

08 especies de carnívoras: Brachyplalystoma flavicans, Arapaima gigas, Pseudoplatystoma fasciatum, Cichla monoculus, Pellona sp; Plagiosum squamosissimus, Brachiplatystoma sp, y Hoplias malabaricus.

En los especímenes muestreados del río A mazonas se tiene: 03 especies de iliófagos Hemiodus sp, Prochilodus nigricans, Acestrorhynchus falcirostres;

04 especies de omnívoras: Mylossoma aureum, Triporteus elongatus, Brycon milanopterum, Cichlasoma sp;

04 especies de carnívoras: Serrasalmus rhonibeus, Hoplias malabaricus, Pseudoplatystoma fasciatum, Pseudoplatystoma tigrinum.

En total se han analizado 26 especies provenientes de tres ríos: Nanay, U cayali y A mazonas.

\section{C aracterísticas de M etales Bioacuinulables}

La mayoría de especies analizadas muestran concentración bajas con algunas excepciones como el caso de Psectogaster amazónica para el río $\mathrm{N}$ anay, Brachyplatystoma flavicans para el río U cayali, este resultado se obtiene teniendo como referencia las concentraciones máximas permitidas en algunos países que se muestran a continuación.

\section{Plomo}

El plomo al igual que el mercurio entra al sistema acuático, vía escurrimiento y de la atmósfera. El plomo en los ríos podría ser insoluble si está 
adherido a partículas orgánicas o inorgánicas, o soluble a la forma de quelatos 0 complejos inorgánicos.

Las aguas superficiales no contaminadas no sobrepasan de $0.1 \mathrm{mg} / \mathrm{l}$. Algunas especies de plantas tienen una gran tolerancia para el plomo que concentran a partir del suelo, formando complejos con las substancias húmicas. La vida acuática puede perturbarse a partir de $0.1 \mathrm{mg} / \mathrm{l}$. Pudiendo los efectos tóxicos manifestarse en los peces a partir de un $1 \mathrm{mg} / \mathrm{l}$. No obstante, la acción tóxica es variable según las especies y el grado de mineralización del agua.

\section{Cobre}

EI cobre además de las vías antes mencionada para los demás metales, se encuentra en el agua por desintegración orgánica principalmente de insectos donde forma parte de la sangre de los mismos en la forma de globina denominado Wemocianina. Puede también encontrarse como trazas, es decir inferior a 1 $\mathrm{mg} / \mathrm{l}$., en ciertas aguas naturales, superiores a este límite como toxicidad relativa se enncuentran como aportes en la alimentación, sin embargo el sabor metálico y astringente de sus sales aparecen en dosis de 4 a $5 \mathrm{mgll}$.

Salvo para algunas especies (Salinonidos), no parece que los contenidos inferiores a $1 \mathrm{mg} / \mathrm{l}$. sean tóxicos para los peces.

Por contra, la vida acuática puede perturbarce con dosis inferiores; pero las condiciones de tóxicidad varian según las especies y la composición del agua (oxígeno disuel to, anhídrido carbónico, temperatura, calcio y magnesio, etc.)

\section{MATERIALES Y METODOS}

Se han empleado diferentes métodos dependiendo de las actividades programadas, tanto en la zona de muestreo como en laboratorios, así tenemos:

\section{M uestreo}

Los muestreos se realizaron en horas de la mañana debido a la mayor oferta de pescado en los lugares de desembarque y en los centros de abasto los lugares de mayor frecuencia de muestreo fueron: el mercado de Belén para especímenes provenientes del río U cayali y Amazonas, mientras que el mercadillo de Bellavista para los especímenes provenientes del río Nanay, en este lugar a veces se ha muestreado en horas de la tarde. El muestreo abarcó las épocas de creciente y vaciante, es decir un año.

Las muestras fueron revisadas cuidadosamente, tratando de que fueran frescas ( $N$ anay y A mazonas) o bién refrigeradas ( $U$ cayali), que permitan su transporte al laboratorio de Quistococha del IIAP, y a los laboratorios de la UNAP. 
A simismo, se ha tratado de que los peces muestreados sean adultos y que ocupen los diferentes niveles tróficos.

\section{Técnicas de M uestreo}

Los especímenes fueron identificados taxonómicamente antes de ser seccionados. Para los análisis se emplearon tejido muscular de la región dorsal, desechándose el resto, este tejido se tritura hasta obtener una masa uniforme, luego de calentado y hervida la muestra en agua destilada, se realiza el filtrado con la muestra fría y con la solución obtenida se analiza la concentración de metales. Siguiendo el $M$ dtodo Complexométrico recomendado por $M$ erck, en el caso de cationes como plomo y Mercurio, se hicieron análisis de comprobación por Absorción Atómica en los laboratorios de la Dirección Tdcnica de Salud A mbiental

(DITESA). Se han determinado M ercurio, Plomo, Cadmio y Cobre (Cu).

\section{RESULTADOS}

A continuación se muestran el número de especies estudiadas y las concentraciones de los metales pesados bioacumulables encontrados durante el estudio. análisis.

L as cantidades son promedios de los especímenes empleados para cada

\section{Concentraciones máximas en ppm permisibles de metales pesados en peces en algunos países americanos.}

\begin{tabular}{|l|l|l|l|l|}
\hline País & M ercurio & Plomo & Cadmio & Cobre \\
\hline Brasil & 0.5 & - & - & - \\
Canadá & 0.5 & 0.5 & - & - \\
Chile & - & 2.0 & 0.05 & 10.0 \\
& EE.UU & 0.5 & - & -- \\
Venezuela & 0.5 & 2.0 & 0.5 & 10.0 \\
\hline
\end{tabular}

Fuente: Complilation of legal limits for harduoz substances in fish and fishery FA OFisheries Circular No 764-Roma (1983). 


\section{PECES PROCEDENTES DEL RIO NANAY}

\begin{tabular}{|c|c|c|c|c|}
\hline MUESTRA (NV/NC) & $\begin{array}{l}\text { Cadmio } \\
\text { ppm }\end{array}$ & $\begin{array}{r}\text { Plomo } \\
\text { ppm }\end{array}$ & $\begin{array}{c}\text { Mereurio } \\
\text { ppm }\end{array}$ & $\begin{array}{r}\text { Cobre } \\
\text { ppm }\end{array}$ \\
\hline 01 BOQUICHICO & 0.0226 & 0.5220 & 0.032 & 0.003 \\
\hline $\begin{array}{l}02 \text { SARDINA } \\
\text { Triportheus elangatus }\end{array}$ & 0.0459 & 0.0120 & 0.001 & 0.001 \\
\hline $\begin{array}{l}\text { 17porineus elongatus } \\
03 \text { PALOMETA } \\
\text { Mylossoma aureum }\end{array}$ & 0.003312 & 0.003315 & 0.00 & 0.005 \\
\hline $\begin{array}{l}04 \text { TUCUNARE } \\
\text { Cichla monaculus }\end{array}$ & 0.0022 & 0.00828 & 0.0009 & 0.0002 \\
\hline $\begin{array}{l}05 \text { LLAMBINA } \\
\text { Potamorhina altamazónica }\end{array}$ & 0.0067 & 0.0145 & 0.0024 & 0.002 \\
\hline $\begin{array}{l}06 \text { RACTACARA } \\
\text { Curimatella sp. }\end{array}$ & 0.0034 & 0.0024 & 0.00048 & 0.0003 \\
\hline $\begin{array}{l}\text { 07 RACTACARA } \\
\text { Curimatella sp. }\end{array}$ & 0,0019 & 0.0045 & 0.00048 & 0.0003 \\
\hline $\begin{array}{l}08 \text { YULILLA } \\
\text { Hemiodus sp. }\end{array}$ & 0.0730 & 0.0300 & 0.00048 & 0.0003 \\
\hline $\begin{array}{l}09 \text { LISA } \\
\text { Schizodon trifasciatus. }\end{array}$ & 0.0067 & 0.0176 & 0.0011 & 0.0001 \\
\hline $\begin{array}{l}10 \text { LLAMBINA } \\
\text { Potamorhina sp. }\end{array}$ & 0.0048 & 0.0332 & 0.0023 & 0.0001 \\
\hline $\begin{array}{l}11 \text { RACTACARA } \\
\text { Psectogaster amazúnico }\end{array}$ & 1.1 & 0.0075 & 0.2 & 0.25 \\
\hline
\end{tabular}

PECES PROCEDENTES DEL RIO UCAYALI

\begin{tabular}{|c|c|c|c|c|}
\hline ESPECIE (NV/NC) & $\begin{array}{l}\text { Cadmio } \\
\mathrm{ppm}\end{array}$ & $\begin{array}{l}\text { Cobre } \\
\text { ppm }\end{array}$ & $\begin{array}{l}\text { Mercurio } \\
\mathrm{ppm}\end{array}$ & $\begin{array}{l}\text { Plomo } \\
\mathrm{ppm}\end{array}$ \\
\hline 01 LLAMBINA & 0.009 & 0.332 & 0.05 & 0.01 \\
\hline $\begin{array}{l}02 \text { SABALO } \\
\text { Brycon melanopterum } \\
03 \text { PACO }\end{array}$ & 0.071 & 0.786 & 0.11 & 0.01 \\
\hline $\begin{array}{l}\text { Plaractus brachypomus } \\
04 \text { LISA COLORADA }\end{array}$ & 0.004 & 0.862 & 0.03 & $0.01^{*}$ \\
\hline $\begin{array}{l}\text { Leporinas fasciatus } \\
\text { 05 MAPARATE }\end{array}$ & 0.089 & 0.420 & 0.06 & $0.01^{*}$ \\
\hline $\begin{array}{l}\text { Hypophthalmus edentatus } \\
06 \text { BOQUICHICO }\end{array}$ & 0.064 & 0.526 & 0.22 & 0.01 \\
\hline $\begin{array}{l}\text { Prochiladus nigricans } \\
\text { 07 DORADO }\end{array}$ & 0.014 & 0.428 & 0.05 & 0.01 \\
\hline $\begin{array}{l}\text { Brachyplatystoma favicans } \\
08 \text { GAMITANA }\end{array}$ & 0.0908 & 0.792 & 0.69 & 0.01 \\
\hline $\begin{array}{l}\text { Colossoma macropomam } \\
09 \mathrm{PAICHE}\end{array}$ & 0.069 & 0.346 & 0.07 & 0.01 \\
\hline $\begin{array}{l}\text { Arupaima gigas } \\
10 \text { DONCEL LA }\end{array}$ & 0.093 & 0.684 & 0.28 & 0.01 \\
\hline $\begin{array}{l}\text { Pseudoplatystoma fasciatum } \\
11 \text { PALOMETA }\end{array}$ & 0.081 & 0.692 & 0.12 & 0.01 \\
\hline 12 Lysassama aureum & 0.001 & 0.191 & 0.01 & 0.01 \\
\hline $\begin{array}{l}\text { Schizodom trifasciatus } \\
13 \text { SARDINA }\end{array}$ & 0.082 & 0.222 & 0.04 & 0.01 \\
\hline $\begin{array}{l}\text { Triporteus angulatus } \\
\text { ThKA }\end{array}$ & 0.004 & 0.106 & 0.10 & 0.01 \\
\hline
\end{tabular}




\begin{tabular}{|c|c|c|c|c|}
\hline ESPECIE (NV/NC) & $\begin{array}{l}\text { Cadmio(Cd) } \\
\text { ppm }\end{array}$ & $\begin{array}{l}\text { Cobre }(\mathrm{Cu}) \\
\mathrm{ppm}\end{array}$ & $\begin{array}{l}\text { Mercurio }(\mathrm{Hg}) \\
\mathrm{ppm}\end{array}$ & $\begin{array}{l}\text { Plomo (Pb) } \\
\text { ppm }\end{array}$ \\
\hline \multirow{6}{*}{$\begin{array}{l}14 \text { TUCUNARE } \\
\text { Cichla monoculus } \\
15 \text { ASNA NAHUI } \\
\text { Pellona sp } \\
16 \text { YULLLLA } \\
\text { Hemiodius sp } \\
17 \text { CORVINA } \\
\text { Plagiosum squamosissimus } \\
18 \text { MANITOA } \\
\text { Brachiplatystoma sp } \\
19 \text { FASACO } \\
\text { Hoplias malabaricus }\end{array}$} & 0.002 & 0.643 & 0.01 & $0.01^{*}$ \\
\hline & 0.004 & 0.226 & 0.19 & 0.01 \\
\hline & 0.008 & 0.416 & 0.01 & 0.01 \\
\hline & 0.092 & 0.148 & 0.18 & 0.01 \\
\hline & 0.012 & 0.214 & 0.35 & 0.01 \\
\hline & 0.019 & 0.2428 & 0.12 & 0.01 \\
\hline
\end{tabular}

- Especímenes juveniles

NV Nonthe Vulgar

NC Nombre cientifico

ppm Partes por millon

\section{PECES PROCEDENTES DEL RIO AMAZONAS}

\begin{tabular}{|c|c|c|c|c|c|}
\hline ESPECIE (NV/NC) & $\begin{array}{l}\text { Cadmio } \\
\mathrm{ppm}\end{array}$ & $\begin{array}{c}\text { Cobre } \\
\text { ppm }\end{array}$ & $\begin{array}{c}\text { Fierro } \\
\text { ppm }\end{array}$ & $\begin{array}{c}\text { Mercurio } \\
\text { ppm }\end{array}$ & $\begin{array}{l}\text { Plomo } \\
\text { ppm }\end{array}$ \\
\hline 01 PAN̂A BLANCA & & & & & \\
\hline $\begin{array}{l}\text { Serrasalmis rhombeas } \\
02 \text { PALOMETA }\end{array}$ & 0.007 & 4.55 & 8.75 & 0.14 & 0.008 \\
\hline Mylossama aureum & 0.006 & 2.80 & 5.5 & 0.12 & 0.01 \\
\hline 03 SARDINA & & & & & \\
\hline $\begin{array}{l}\text { Triportheus elongatus } \\
04 \text { FASACO }\end{array}$ & 0.0018 & 0.75 & 0.75 & 0.08 & 0.017 \\
\hline $\begin{array}{l}\text { Hoplias malabaricus } \\
05 \text { BUJURQUI }\end{array}$ & 0.02 & 3.2 & 10.3 & 0.1 & 0.006 \\
\hline $\begin{array}{l}\text { Satanoperca jurupari } \\
\text { 06 PEZ ZORRO }\end{array}$ & 0.006 & 2.60 & 0.04 & 0.04 & 0.01 \\
\hline $\begin{array}{l}\text { Acestrorhynchus } \\
\text { falcinostris } \\
07 \text { SABALO }\end{array}$ & 0.014 & 0.48 & 7.15 & 0.07 & 0.01 \\
\hline $\begin{array}{l}\text { Byycan melanopterum } \\
08 \text { YULILLA }\end{array}$ & 0.06 & 4.7 & 9.3 & 0.17 & 0.005 \\
\hline $\begin{array}{l}\text { Hemiodiss sp. } \\
09 \text { BOQUICHICO }\end{array}$ & 0.04 & 5.6 & 16.53 & 0.01 & 0.0013 \\
\hline $\begin{array}{l}\text { Prachilodus nigricans } \\
10 \text { ZUNGARO (doncella) }\end{array}$ & 0.006 & 4.8 & 12.25 & 0.05 & 0.013 \\
\hline $\begin{array}{l}\text { Pseudoplatystoma } \\
\text { fasciatum } \\
11 \text { ZUNGARO TIGRE }\end{array}$ & 0.03 & 3.6 & 9.77 & 0.26 & 0.019 \\
\hline $\begin{array}{l}\text { Psenuloplatystowa } \\
\text { tigrinus }\end{array}$ & 0.035 & 3.8 & 10.17 & 0.32 & 0.007 \\
\hline
\end{tabular}


ANALISIS DE METALES BIOACUMULABLES EN PECES PROCEDENTES DE LORETO EN PPM

\begin{tabular}{|c|c|c|c|c|}
\hline ESPECIE / AÑO & Mercurio & Cobre & Cadmio & Plomo \\
\hline \multicolumn{5}{|l|}{1986} \\
\hline$\overline{\text { DORADO (Pastaza) }}$ & $1.82 *$ & & & 1.062 \\
\hline $\begin{array}{l}\text { Brachyplatystama fasciatumbs } \\
\text { BAGRE (Corrientes) }\end{array}$ & $1.74^{*}$ & 3.4 & 0.02 & 1.092 \\
\hline $\begin{array}{l}\text { Pimelodis sp. } \\
\text { ASNAN̄AHUI (Corriente) }\end{array}$ & 0.07 & 3.3 & $0.03+$ & 0.663 \\
\hline $\begin{array}{l}\text { Pellona sp. } \\
1987 \\
\text { SARDINA (feb) }\end{array}$ & $0.46+$ & $13.4^{*}$ & 0.02 & 0.181 \\
\hline Triporireus sp. & $0.51 *$ & $7.8+$ & 0.01 & 0.830 \\
\hline MÓJARA & 0.24 & $10.5 *$ & $0.04+$ & 0.121 \\
\hline \multicolumn{5}{|l|}{$\begin{array}{l}\text { Tetraganoptents argenteus } \\
\text { BAGRE }\end{array}$} \\
\hline $\begin{array}{l}\text { Pimelodeus sp. } \\
\text { SHIRIPIRA }\end{array}$ & $0.42+$ & 4.7 & 0.00 & 0.331 \\
\hline $\begin{array}{l}\text { Sombim lima } \\
\text { MOTA (marzo) }\end{array}$ & $0.38+$ & 7.3 & 0.01 & 0.096 \\
\hline $\begin{array}{l}\text { Brachyplatystoma sp. } \\
\text { ASNANAHUI }\end{array}$ & $0.91 *$ & 2.3 & 0.00 & 0.963 \\
\hline $\begin{array}{l}\text { Pellona sp. } \\
\text { LISA (mayo) }\end{array}$ & $0.51 *$ & 2.6 & 0.00 & 0.246 \\
\hline $\begin{array}{l}\text { Schizodon sp. } \\
\text { ANGUILA }\end{array}$ & 0.14 & $14.3^{*}$ & 0.00 & 0.468 \\
\hline $\begin{array}{l}\text { Electrophorus eléctricus } \\
\text { MAPARATE }\end{array}$ & 0.37 & 5.6 & $0.05^{*}$ & 0.194 \\
\hline $\begin{array}{l}\text { Hypophthalmus edentatus } \\
\text { ASNAÑAHUI }\end{array}$ & $0.61 *$ & 5.0 & 0.00 & 0.885 \\
\hline $\begin{array}{l}\text { Pellona sp. } \\
\text { SARDINA (julio) }\end{array}$ & $0.40+$ & 3.8 & 0.00 & 0.366 \\
\hline $\begin{array}{l}\text { Triporlews sp. } \\
1988\end{array}$ & 0.01 & 0.24 & $0.12 \bullet$ & 0.289 \\
\hline$\overline{\mathrm{DOR}} \mathrm{ADO}$ & & & & \\
\hline Brachyplatysioma sp. & $0.61 *$ & - & & 0.962 \\
\hline
\end{tabular}

- Sobrepasan limites

$+\quad$ Cercanos al línite.

\section{DISCUSION}

L os resultados encontrados nos muestran concentraciones bajo los límites permisibles para consumo humano, con muy pocas excepciones a los valores reportados por Cánepa, et al (1987) para el río Corrientes, así como los reportados por Pezo (1986) para el río Pastaza especialmente en la especie Brachyplatystoma filamentosum, tal como se muestra a continuación : 
Los resultados muestran que hay poca diferencia en concentración de metales pesados entre los tres niveles tróficos, iliófago, omnívoro y carnívoro, presentando las mayores concentraciones los carnívoros, entre los dos primeros niveles es aún menor la diferencia es posible que los iliófagos se contaminen al filtrar los detritus orgánicos en los lechos de ríos o cochas principalmente en ambientes lénticos, por otra parte puede considerarse concentraciones normales y naturales teniendo en cuenta el origen de los ríos que forman la cuenca, los diferentes tipos de suelos que cruza en su recorrido, cationes compuestos orgánicos e inorgánicos, sólidos en suspensión, etc. que le dan características propias a cada río.

Estos resultados a su vez pueden servir para calcular la capacidad de soporte del medio ambiente acuático, es decir cuanto puede recibir y reciclar sin producir daños a los consumidores de primer o segundo orden; indudablemente se requiere realizar muchos estudios para determinar la capacidad del pez amazónico de desintoxicarse.

En el estudio realizado por Cánepa J, et al (1987) reportan concentraciones al tas de metales en lechos del río Corrientes y tributarios, lo cual demuestra que la fuente de contaminación de los peces iliófagos, principalmente estaría en el bentos, y luego pasa a los siguientes niveles tróficos hasta llegar al hombre, en este nivel no se ha realizado ningún estudio hasta el momento o por lo menos no existen reportes sobre el tema en la amazonía.

\section{CONCLUSIONES}

Los peces estudiados pertenecen a los tres niveles tróficos, iliófagos, omnívoros y carnívoros para cada uno de los ríos.

Los peces estudiados en términos generales no presentan concentraciones que pueden considerarse peligroso para el consumo humano.

Las variaciones del ciclo hidrológico de los ríos no influyen en las concentraciones de los metales pesados bioacumulables en peces.

La mayor cantidad de especies estudiadas fueron los provenientes del río Ucayali.

La mayoría de los especímenes estudiados fueron de estado adulto de ambos sexos.

\section{RECOMENDACIONES}

Realizar estudios similares en Pucall pa u otras ciudades de la amazonía. Establecer un programa de monitoreo a fin de conocer las posibles variaciones de las concentraciones de los metales bioacumulables en las especies ícticas de mayor consumo local yio regional. 
Estudiar las fuentes de contaminación de Mercurio y Plomo en el río Pastaza.

\section{BIBLIOGRAFIA}

ARRIGNTON, J. "Ecología y Piscicultura de Aguas dulces. Ediciones $M$ undiPrensa-M adrid-365 $\mathrm{p}$.

CANEPA,J . (1982) "Ecología del sábalo cola roja Brycon erythropterum en el sistema de laguna Supay y aledaños, Jenaro Herrera (Tesis) 122 p.

CANEPA, J. R. PEZO, H. PAREDES, (1987) "Estudio Hidrobiológico del Río Corrientes-IIAP, Iquitos $70 \mathrm{p}$.

FAO (1981) "Manual de Métodos de Investigación del Medio Ambiente A cuático, Parte 4ta. Base para Elección de Ensayos Biológicos para evaluar la contaminación M arina D oc.T ec. Pesca (164) 34 p.

FAO (1975) "Curso de Capacitación FA O/sida sobre contaminación de las aguas del mar en relación con la protección de los recursos vivos. Gotemburgo Suecia. Supl. 1:328 p.

GOULDING, M. (1981) "The fishes and the forest University of California Press Berkeley Los A ngeles, L ondon.

MACO,J., R. PEZO, J. CANEPA, (1985) "Efectos de la Contaminación Ambiental por Actividades Petroleras. Fase Producción (SCAPZT) Iquitos $22 \mathrm{p}$.

NAUEN, E.E. (1983) "Compilation of legal limits for hazardous substances in fish and fishery products, FA O, FISH; (764) 102 p.

PEZO R, J. CANEPA, J. MACO, (1985) "Efectos de la Contaminación A mbiental por A ctividades Petroleras sobre la Flora y Fauna (SCA PZT) Iquitos $18 \mathrm{p}$.

PESSON, P. (1979) "La contaminación de las A guas Continentales". Ediciones M undi-Prensa, M adrid, $335 \mathrm{p}$. 